\title{
XLI. On vanadium
}

\section{Berzelius}

To cite this article: M. Berzelius (1831) XLI. On vanadium , Philosophical Magazine Series 2, 10:59, 321-337, DOI: 10.1080/14786443108675553

To link to this article: http://dx.doi.org/10.1080/14786443108675553

曲 Published online: 14 Jul 2009.

Submit your article to this journal

山 Article views: 10

Q View related articles $₫$ 


\section{PHILOSOPHICAL MAGAZINE}

A N D

\section{ANNALS OF PHILOSOPHY.}

[NEW SERIES.]

NOVEMBER 1831.

\section{On Vanadium. By M. Berzelius*.}

V ANADIUM was discovered in the year 1830 by Sefström, in a Swedish iron, remarkable for its ductility, obtained from the iron mine of Jaberg, not far from Jönköping in Sweden. The name of this metal is derived from that of Vanadis, a Scandinavian divinity. It is not yet known under what form, or in what state of combination, vanadium occurs in the ore of Jaberg. It is also found in Mexico, in a lead mine at Zimapan. Del Rio, who analysed this mineral in 1801, announced the discovery of a new metal in it, which he called Erythronium; but the same mineral having soon afterwards been analysed by Collet Descotils, he asserted that erythronium was merely impure chromium. Del Rio himself adopted the opinion of the French chemist, and considered the mineral as a subchromate of lead; thus the metal, so near being discovered, remained thirty years unknown to chemists. Since the discovery of vanadium by Sefström, Wohler has ascertained that the mineral of Zimapan contains vanadic and not chromic acid.

I have had an opportunity of studying the properties of this metal, and those of its combinations, by means of specimens presented to me by M. Sefström for that purpose.

M. Sefström having ascertained that the finery cinder of the cast-iron of Jaberg contained more vanadium than the iron itself, made use of it to obtain the metal, which occurs in it in the state of vanadic acid. For this purpose he uses the following process: the finery cinder is powdered and mixed with

* Traité de Chimic, tom. iv. p. 649. See also the present volume of Phil. Mag. and Annals, p. 151, 157, and 209 ; and the Misclloneous Articles in the present Number.

N. S. Vol. 10. No. 59. Nor. 1831 . 'T nitre 
nitre and carbonate of soda, in the proportions of one part of cinder, one of nitre and two parts of carbonate; this mixture is strongly calcined for an hour. The soluble portion of the powdered mass is dissolved by boiling water, the solution is filtered, and the excess of alkali saturated with nitric acid and afterwards precipitated with muriate of barytes or acetate of lead. The precipitate is vanadate of barytes or lead, containing also some phosphate of barytes or lead, silica, zircon, and alumina. Whilst it is still moist, it is to be decomposed by concentrated sulphuric acid; the solution immediately becomes of a deep red colour, and after having digested the mixture for half an hour, alcohol is added to it, and it is again digested; xther is then formed, and the vanadic acid is reduced to the state of salifiable oxide, the solution of which is blue; and when it begins to assume a syrupy consistence, it is mixed in a platina crucible with a little fluoric acid to separate a portion of silica, which it is almost impossible to get rid of in any other manner; the evaporation is continued over the naked fire, and the sulphuric acid is at last expelled at a red heat. The residue is impure vanadic acid. It is fused with nitre, added in small portions at a time. The vanadic acid combines with the potash and expels the nitric acid, and nitre is added until it is found that on cooling a small portion of the mass it ceases to be red. The alkaline carbonates may be employed ; but when nitre is used, the zircon and alumina remain less acted upon, when the vanadate of potash is dissolved. The mass is afterwards dissolved in water, and after filtration the residue is slightly washed; it still contains vanadium, and ought not to be thrown away. A piece of sal ammoniac, larger than can be dissolved by it, is to be put into the filtered liquor. As this salt dissolves, a white pulverulent precipitate is formed, which is vanadate of ammonia, insoluble in a saturated solution of sal ammoniac. The phosphate of ammonia remains dissolved; but when the solution is alkaline, which happens when a carbonate is employed to dissolve the vanadic acid, insoluble subphosphate of ammonia always precipitates. The vanadate of ammonia ought to be washed, first with a solution of sal ammoniac, and afterwards, to remove the sal ammoniac, with alcohol of 0.86 . It is to be again dissolved in boiling water, mixed with a little ammonia, filtered and left to crystallize. It is from this salt that vanadic acid and oxide are afterwards obtained, by heating it gently in open vessels to procure the former, and in closed vessels to prepare the latter.

The residue which has been mentioned is a compound of vanadic acid, alumina, zirconia and silica. The vanadium is extracted 
extracted from it by means of an alkaline hydrosulphuret, by fusing the residue with sulphur and carbonate of potash. Sulphovanadate of potash is formed, from which sulphuret of vanadium may be precipitated by sulphuric acid.

Vanadium is very difficult of reduction by the usual methods, that is to say, by heating the oxide in a charcoal crucible; for it is reduced only at the places in which it is in immediate contact with the charcoal, and the interior is a suboxide, as infusible as the metal itself at the temperature at which manganese undergoes fusion.

With potassium the reduction is easy; pieces of vanadic acid, which have been previously fused, are to be mixed with pieces of potassium of equal bulk in a porcelain crucible; the cover is to be well fastened on, and the crucible is to be beated with a spirit-lamp. The reduction occurs almost instantaneously with a kind of detonation. The crucible, when cold, is to be put into water to dissolve the potash, and the reduced vanadium is to be collected on a filter; it is obtained in the state of a black powder, which shines in the sun, and takes a grayish metallic lustre underthe burnisher. But in this way a true idea of the aspect of the metal is no more obtained, than of that of gold precipitated from solution by the salts of iron.

When the method discovered by $M$. Rose for the reduction of titanium is employed to reduce vanadium, the experiment succeeds more completely than with potassium. For this purpose, chloride of vanadium is prepared, by passing a current of dry chlorine over a mixture of vanadic acid and very dry charcoal. This chloride is a volatile fuming fluid, and it is to be introduced into a glass bulb blown on a barometer tube; a current of dry ammoniacal gas is passed through the tube until the chloride is entirely saturated. Sal ammoniac sublimes, which may be expelled from the tube by another spirit-lamp. The reduced vanadium remains in the bulb, and an inconsiderable portion is reduced at that part of the tube which is kept hot. On cutting the bulb afterwards in two, the vanadium is found in the state of a silvery white stratum, which on the side next the glass reflects like a mirror, and is white like polished steel. If water and atmospheric air are not entirely excluded, a small quantity of black powder is found in the middle of the mass; this is suboxide of vanadium, and is easily detached.

Vanadium is white, and when its surface is polished it resembles silver considerably, or molybdenum, which of all metals it is most like. It is not ductile, and is easily reduced to a powder of an iron-gray colour. I have not enough of it, nor are my specimens in a convenient form, to determine its \& $\mathrm{T} 2$

specific 
specific gravity. It is a good conductor of electricity, and strongly negative to zinc. The powder of vanadium, obtained by its reduction with potassium, takes fire at a heat below redness, burns without energy, and leaves a black unfused oxide. Vanadium dissolves readily in nitric acid and in aqua regia; the solution has a fine blue colour. The sulphuric, muriatic, and fluoric acids do not attack it at all, even when they are concentrated and boiling. It is not oxidized by the alkaline hydrates, and it may be heated with them to redness without undergoing any alteration if the air be excluded. 'The solution of vanadic oxide in the acids, or of vanadic acid in an excess of caustic potash, does not give metallic vanadium by zinc.

Oxides of Vanadium. - Of this metal there are three compounds with oxygen :-

1st, Suboxide of Vanadium.-It is obtained by reducing vanadic acid by hydrogen gas at a red heat, or by fusing vanadic acid in a cavity made in charcoal. In the first mode, the suboxide preserves the form and lustre of the crystalline facets of the acid, but it becomes black; by the latter process a coherent mass is obtained, which is easily reducible to powder, possesses a semimetallic lustre and the colour of plumbago. Hydrogen passed over the suboxide does not decompose it at the highest temperature which can be imparted to it in a porcelain tube heated by a small wind-furnace. This suboxide, by whatever process obtained, provided it be coherent, is a good conductor of electricity, and infinitely exceeds copper, and even gold and platina, as a negative electromotor.

It has not hitherto been combined with other bodies, or with acids or bases. That which is reduced by hydrogen gas gradually oxidizes in the air, but without any alteration of appearance; and the lower the temperature at which the oxide is formed, the more readily oxidation occurs. Its oxidation is apparent by throwing it into water, which becomes of a fine green colour by dissolving a compound presently to be treated of. When heated in the air, it takes fire and burns, leaving an unfused black residue. Chlorine gas converts it into chloride and vanadic acid. It is composed of 89.538 parts of vanadium, and 10.862 parts of oxygen; 100 parts of the former are combined with 11.6843 parts of the latter.

2ndly, Oxide of Vanadium.-Vanadate of ammonia cannot be employed in the preparation of this oxide in the same way as the molybdate and tungstate of ammonia, which yield the oxides of their metals when they are heated. The oxygenated compound of vanadium obtained by this method, contains the three degrees of oxidation of this metal. In order to obtain 
pure oxide of vanadium in the dry way, 9.5 parts of suboxide of vanadium are to be mixed with 11.5 parts of vanadic acid, and the mixture is to be heated to whiteness, in an atmosphere of carbonic acid gas. In the moist way, it may be obtained by precipitating a blue vanadic salt, previously treated by sulphuretted hydrogen, sugar or alcohol, in order to destroy all the vanadic acid which it may contain. This solution is to be precipitated by carbonate of soda, added slightly in excess. A grayish white precipitate is formed, which is collected on a filter and washed, without the contact of the air. It is to be pressed between folds of filtering paper and dried in vacuo. It is gray, inclining to brown: it is hydrated oxide of vanadium, sontetimes containing traces of carbonic acid. When heated to redness in vacuo, it yields water and leaves the oxide in the state of a black powder, which does not blue litmus paper that has been previously reddened. Oxide of vanadium is not fusible at the temperature at which glass softens. It is insoluble in water, but if it remains long in it the water becomes gradually green, in consequence of increased oxidation. 'The hydrate rapidly oxidizes in the air, and becomes first brown and afterwards green; when dried it is black: it will be again noticed. Oxide of vanadium which has been heated, dissolves slowly but completely in acids; the solution is blue, and the oxide acts as a base; but it combines with bases and forms salts, which may be called vanadites. The alkaline carbonates dissolve it; the solution, which is of a deep brown, contains a vanadate and a bicarbonate; the bicarbonates also dissolve it, and assume a blue colour: it appears that this solution contains neutral double carbonate of vanadium and alkali. Oxide of vanadium is composed of $81 \cdot 0.56$ parts of vanadium, and 18.944 of oxygen, or 100 parts of the metal combine with 23.369 parts of oxygen, that is to say, with twice as much as in the suboxide.

3rdly, Vanadic Acid.-This is obtained by exposing vanadate of ammonia to a heat near redness in an open platina crucible, and stirring it occasionally. The vanadate decomposes, becomes at first black, and afterwards, in proportion as it absorbs atmospheric oxygen, of a red brown colour, which, by cooling, becomes gradually pale, and finishes by turning to a rust colour. The finer the sal ammoniac is powdered, the paler is the colour of the acid. The acid thus obtained, when triturated, becomes of the colour of the hydrate of iron, which forms on the surface of the metal immersed in water. It is tasteless and inodorous; it reddens the colour of moistened litmus paper. As soon as it is red hot it fuses. In this state it sustains a white heat without losing 
losing oxygen, if it be preserved from the influence of combustible bodies. When fused it crystallizes on cooling, and then exhibits a phænomenon, which merits observation. It solidifies at a heat which is invisible in daylight ; but the moment that solidification commences, a luminous circle extends from the periphery to the centre, where, owing to latent heat becoming free, the mass remains red hot as long as the crystallization continues. The acid contracts much on solidifying, and is readily detached from the crucible: it is then of a yellowish red colour, and formed entirely of a mass of interlaced crystals. Cavities frequently occur containing small and perfectly regular crystals, the form and size of which may be determined, when opportunity offers of repeating the experiment with about 300 grains. Fused vanadic acid is translucent at the edges, and has a yellowish colour. When it is impure, or when it has been in part reduced to the state of oxide, it does not crystallize; but at the moment of its solidification, excrescences are produced in the form of cauliflowers, and the solidified mass is blackish. If the acid contains a very small quantity of oxide, it crystallizes, but afterwards assumes a violet colour. Vanadic acid is not a conductor of electricity. It is slightly soluble in water, to which it imparts a bright yellow colour. If the pulverulent acid be put into water and well stirred, it mixes with it so as to produce a turbid fluid of a yellow colour, which does not become clear for several days : 1000 parts of boiling water scarcely dissolve one part of vanadic acid, but the cooled solution remains transparent. The acid is deposited by evaporation in the form of red concentric rings. The last portion gives yellowish microscopic crystals, but they become green when heated. It is a compound of vanadic oxide and acid, produced apparently by the influence of dust floating in the air; a phænomenon similar to the partial reduction of a solution of oxymanganic acid, which is attributed to this cause. It is in general impossible to crystallize vanadic acid in the humid way, and it is equally so to extract it in an isolated state from a solution, because it combines equally with acids and bases. It is easily reduced to the state of oxide, especially under the influence of an acid; red nitric acid, sulphurous acid, several vegetable acids, especially the oxalic and tartaric, alcohol, sugar, \&c. effect this reduction at a moderate temperature. Muriatic acid dissolves and becomes of an orange colour; but soon afterwards chlorine is disengaged, and the solution then possesses the property of dissolving gold and platina. Vanadic acid, fused on charcoal by the blowpipe, leaves a coherent mass, of the colour of plumbago, which is the suboxide of vanadium: 
vanadium: with the phosphate of ammonia and soda it gives a fine green colour to glass, which appears brown while it is hot. The blue colour of the salts of vanadium cannot be produced, even on adding metallic tin to the flux. With borax it also gives a green glass. In this reaction vanadium resembles chromium, but the green colour produced by the former may be changed to yellow by the oxidating flame, which does not happen with chromium. This change is easily effected, especially with the glass of borax. With carbonate of soda it is not reduced to the metallic state. Vanadic acid is composed of 74.0449 parts of vanadium and 25.9551 of oxygen, that is to say, 35.0533 of the latter, and 100 of the former; consequently the metal is combined with three times as much oxygen as in the suboxide. Its saturating capacity is equal to one third of the quantity of oxygen which it contains, that is to say, 8.6517 .

4thly, Intermediate Oxides of Vanadium.-We have seen that the suboxide and oxide of vanadium, when exposed to the influence of the air, acquire the property of colouring water green. The vanadic oxide and acid combine together in different proportions; two of these compounds have the property of forming with water a solution of a fine green colour. Other compounds are purple and orange coloured. They pass, by the influence of the air, from one degree of oxidation to a higher one.

a. Purple Oxide.- If vanadic acid be kept for twenty-four hours in a badly corked bottle, and water be then added to it, it becomes of a green colour. The mass is then to be poured upon a filter, and when the green liquor is filtered a fresh portion of water is added; the fluid which then filters is much deeper coloured and brownish; a fresh quantity of water assumes a fine purple colour, and when the washings have been thus continued for some time, the water passes through colourless. The residue exposed for some time to the air acquires the property of reproducing the phænomena which have been described, and eventually a new purple liquid is obtained. This solution holds but little matter in solution; it may be preserved in a full bottle hermetically sealed, but on exposure to the air it soon becomes green and afterwards yellow. The vanadic acid appears to be combined with the greatest quantity of oxide of vanadium that it is capable of rendering soluble. It may be called a subvanadate of vanadium.

b. Vanadate of Vanadium. - If hydrate of vanadium be allowed to dry in the air, and then digested in a very small quantity 
quantity of water, it becomes of a green colour, which is beautiful, but so deep that the solution appeares opake. The solution, when filtered and evaporated in vacuo, leaves a blackish cracked residue, without any trace of crystallization, and which is completely resoluble in water. This same combination is obtained, when a solution of a neutral salt with a base of oxide of vanadium is mixed with neutral vanadate of potash. If the solutions are moderately concentrated, a great part of the new green compound formed is deposited in the state of deep coloured powder; and if the solution is too dilute to give a precipitate, one is obtained by dissolving sal ammoniac in it. The precipitate is insoluble in absolute alcohol, but it dissolves in alcohol of 0.86 . The solutions of this substance diluted so as to become perfectly transparent, have a very fine green colour. A small quantity of alkali deepens the colour, but does not destroy the green compound. The addition of a caustic alkali in excess occasions in a short time a brown precipitate, which is a vanadate of the aikali added. The carbonates of soda and potash change the green colour to brown, without precipitating anything; an excess of carbonate of ammonia does not destroy the colour. Vanadic oxide, mixed and digested with vanadic acid, forms the same compound, which may also be produced in the dry way by heating an intimate mixture of $10 \frac{\mathrm{I}}{2}$ parts of oxide, and $23 \frac{\mathrm{l}}{\mathrm{T}} \mathrm{D}$ parts of vanadic acid. The mixture fuses, and gives a glass of a deep green colour, the powder of which dissolves gradually in water.

c. Bivanadate of Vanadium.-This compound is obtained by mixing a neutral salt of vanadiun with one of bivanadate of potash; this salt is solid and green like the preceding, the tint of which is deeper; its solution in water is of yellowish green. It is less soluble than the preceding, and is more completely precipitated by sal ammoniac.

d. Supervariadate of Vanadium.-All the purple and green compounds oxidize in the air, especially when they are very dilute. Their colour becomes first greenish yellow, and afterwards orange yellow. By spontaneous evaporation, they yield crystals of a pale orange yellow colour, which lose their water and become green when heated in the fire; 22.5 parts of water dissolve one part of this orange compound, consequently it is much more soluble than vanadic acid alone.

Sulphurets of Vanadium.-The affinity of varadium for sulphur is but weak at moderately high temperatures; it may be mixed with sulphur, and the mixture may be distilled without undergoing combination; and even when it is heated to redness in an atmosphere of sulphur, vanadium is not sulphu- 
retted. Nevertheless, there are several modes of obtaining sulphurets of vanadium; hitherto, only two have been formed, proportional to the oxide and vanadic acid.

1. Sulphuret of Vanadium.-This is obtained in the dry way by exposing suboxide of vanadium to a current of sulphuretted hydrogen at a red beat. Water, hydrogen, and even sulphur are disengaged, and the vanadium is slowly converted into a sulphuret. This sulphuret is black; it becomes compact by pressure; when burnished it has not a metallic lustre. Heated in platina foil, it burns with a blue flame, and leaves upon the platina a circular pellicle, which is translucent, blue at the circumference and purple nearer the sulphuret. Water does not remove this pellicle, but it disappears at a red heat, leaving minute drops of vanadic acid. In this state sulphuret of vanadium is entirely insoluble both in sulphuric and muriatic acids, and in the caustic alkalies. Nitric acid converts it into sulphate of vanadium.

The salts of vanadium are not decomposed by sulphuretted hydrogen, but the hydrate and the salts of vanadium are converted by the hydrosulphurets into sulpho-vanadates, which dissolve in water; the solution has a rich purple colour. Acids poured into these solutions occasion a brown precipitate, which soon subsides and then appears black; it is the sulphuret of vanadium: it may be washed and dried without undergoing any alteration; it dissolves with a purple colour in the alkaline hydro-sulphurets. The alkaline carbonates also dissolve it when boiling, but the colour of the solution is brownish yellow. The sulphuric and muriatic acids do not decompose it, although the liquid from which it has been precipitated retains a bluish tint, in consequence of the decomposition of a small quantity of nascent sulphuret. It is composed of 68.023 parts of vanadium, and 31.977 parts of sulphur.

2. Supersulphuret of Vanadium.-The affinity of sulphur for vanadium is so weak, that when a current of sulphuretted hydrogen is passed into an aqueous solution of vanadic acid, the precipitate is merely oxide of vanadium intimately mixed with sulphur, from which the acids separate the oxide without disengaging sulphuretted hydrogen, and leave the sulphur. To obtain the supersulphuret of vanadium, vanadic acid must be dissolved in an alkaline hydro-sulphuret, or the solution of a neutral vanadate of an alkali must be decomposed by sulphuretted hydrogen, and the sulphuret must be afterwards precipitated by sulphuric or muriatic acid. The colour of the precipitate is brown, but much less deep than that of the preceding sulphuret; and there is this peculiar circumstance attending it, that at the time of the addition of the acid it deN.S. Vol. 10. No. 59. Nov. 1831. $2 \mathrm{U}$ composes 
composes a portion of the supersulphate of vanadium in its nascent state, and much more than of the sulphuret under the same circumstances. The supersulphuret of vanadium may be dried and kept without suffering any alteration; it appears to be black, but the powder is brown. At a high temperature it yields sulphur, and is converted into sulphuret: it dissolves in the same menstrua as the sulphuret, but its solutions have a deep colour resembling that of strong beer. Sulphuric and muriatic acid do not decompose it. It is composed of $58^{* 647}$ parts of vanadium, and $41 \cdot 353$ of sulphur.

Phosphuret of Vanadium.-When vanadium is heated to redness in an atmosphere of phosphorus in vapour, they do not combine; but when phosphate of vanadium is heated to whiteness in a charcoal crucible, it is reduced, and gives a porous, gray, unfused mass, which may be compressed, and has then the colour and lustre of plumbago.

Alloys of Vanadium.- This branch of the history of vanadium remains to be investigated. M. Sefström, who is principally occupied with metallurgic researches, intends to make it the subject of extensive investigation. The experiments which I have had an opportunity of making on the subject prove that vanadium combines readily with other metals; it is sufficient to fuse by the blowpipe on charcoal several metallic vanadates, to reduce them to the state of alloys of vanadium ; but in this case they are deprived of ductility. In experiments upon vanadium, the surface of platina crucibles is often alloyed with vanadium, which does not alter either the colour or the metallic lustre of the platina; but when it is afterwards heated to redness, the alloyed parts are covered with a layer of fused vanadic acid which preserves them from further oxidation. When they are heated to redness, and afterwards washed with potash (and this is repeated five or six times), the vanadium is separated. Neither bisulphate of potash nor borax mixed with nitre when melted in the crucible succeed so well. I have not found that the crucibles were in any way injured.

Salts of Vanadium*.-The salts which contain oxide of vanadium as a base are, with few exceptions, of a superb azure blue colour when in solution. In the solid state and combined with water, they are either of a deep or light blue colour, and sometimes greenish. Without water they are generally brown, and sometimes also green. Both the brown and green salts give blue solutions. Their taste is astringent and rather

* In this translation we have entirely neglected Berzelius's newly invented nomenclature. It has increased the difficulties of the science, without adding one advantage, that we can perceive.-Enir.

sweetish, 
sweetish, like those of iron. The greater number of them are soluble irr water; the caustic alkalies occasion a precipitate, which is at first of a grayish white colour, and which afterwards becomes of a liver brown; an excess of alkali dissolves the precipitate, producing a solution of a brown colour. Ammonia added in excess gives a brown precipitate, and the liquid becomes colourless. The carbonates occasion grayishwhite precipitates: sulphuretted hydrogen does not render them turbid, but the hydrosulphurets occasion a black precipitate, and when added in excess they redissolve it, occasioning a fine purple colour; ferrocyanate of potash occasions a lemon-yellow precipitate, which becomes green in the air. Infusion of galls gives a precipitate of so deep a blue colour that it appears black.

Chloride of Vanadium.-This salt has not been obtained in an anhydrous state: it can only be formed by passing the vapour of chloride of vanadium over a mixture of suboxide of vanadium and charcoal heated to redness : when sulphate of vanadium, dried as much as possible, is mixed with chloride of potassium and fused, the mass contains nearly the whole of the vanadium. It appears that the vanadium is converted into acid at the expense of the sulphuric acid.

Chloride of vanadium (muriate?) is very easily obtained in combination with water. If vanadic acid is dissolved in concentrated muriatic acid and heated, chlorine is evolved; but when it is digested with suboxide of vanadium or with vanadium, chloride is obtained free from chlorine. The same effect is produced by adding to the solution a little sugar, sulphuretted hydrogen, or alcohol. The solution has a fine blue colour. If, on the contrary, concentrated muriatic acid be poured upon oxide of vanadium prepared by calcining vanadate of ammonia in close vessels, a brownish black solution is obtained, and a little chlorine is disengaged, owing to the decomposition of a little vanadic acid. The brown liquid, saturated as perfectly as possible with oxide, suffered to evaporate spontaneously, becomes concentrated to a certain point, but is not rendered dry. Diluted with water, it retains its brown colour; but when evaporated with heat, it becomes gradually completely blue. This change takes place immediately when sulphuric acid is added, even to the concentrated liquid; and in this case neither precipitation nor the evolution of any gas occurs. It appears that the brown chloride differs only from the blue, in being in a different isomeric state, which the sulphuric acid instantly changes. The blue chloride gradually concentrates, and in thin layers it dries, leaving a brown varnish, which is no longer perfectly soluble in water. Evaporated at a mode- 
rate heat, it is entirely converted into this brown mass, which is a subchloride. It gives no appearance of crystallization. Concentrated chloride of vanadium may be mixed with absolute alcohol without precipitation. Ammonia occasions a greenish gray precipitate, which may be washed without dissolving, and which appears to be a subchloride containing ammonia.

Bromide of Vanadium.-This salt resembles the blwe chloride in all respects. Hydrobromic acid dissolves anhydrous oxide of vanadium, the solution is blue. The concentrated bromide, mixed with absolute alcohol, becomes in a few seconds gelatinous, because the alcohol seizes the water; but it becomes liquid in proportion as the alcohol evaporates. When dried it becomes brown, but it redissolves almost entirely in water. Ammonia precipitates a greenish gray double chloride.

Iodide of Vanadium. - Its solution is blue like that of the preceding salts, but it becomes quickly green in the air. By spontaneous evaporation it becomes a semi-fluid brown mass, which when diluted is of a blackish brown colour. Sulphuric acid then disengages iodine. It appears to contain a mixture of vanadate of vanadium and of ioduretted iodide of vanadium. I have not examined it very minutely.

Fluoride of Vanadium. - In solution it is blue, and when dried brown, and redissolves in water. By spontaneous evaporation it becomes greenish and of a syrupy consistence, and greenish crystals are formed. In this state it is soluble in absolute alcohol, which does not restore its original blue colour; sulphuretted hydrogen easily produces this effect. This fluoride combines with the alkaline fluorides, with which it forms salts of a light blue colour, very soluble in water, but not in alcohol.

Fluo-silicate of Vanadium.-The solution is blue; when evaporated at $140^{\circ}$ Fahrenheit it gives a spongy light blue coloured mass. By evaporation it becomes green, and resembles the fluoride.

Cyamuret of Vanadium. - When hydrate of vanadium is treated with hydrocyanic acid, it becomes brown, and it may be washed without becoming green or dissolving. I afterwards treated it with cyanuret of potassium; it dissolved, but the solution left to spontaneous evaporation only gave vanadate of potash, exhaling at the same time the odour of hydrocyanic acid. The ferrocyanate of vanadium precipitates in a bulky mass, of a fine lemon colour with a greenish tint. It is not soluble in diluted acids; by exposure to the air it becomes of a fine green; the perferrocyanate of vanadium precipitates in a gelatinous mass of a yellowish green. 
Sulphate of Vanadium.-This salt is obtained by dissolving vanadic acid or oxide (as obtained by the calcination of vanadate of ammonia) in sulphuric acid, mixed with an equal quantity of water, and by passing sulphuretted hydrogen into the solution diluted with water, in order to reduce the last traces of the vanadic acid dissolved. Oxalic acid may be used for the same purpose; the liquid is to be evaporated until the excess of sulphuric acid begins to concentrate; the salt is then deposited in the form of a transparent crystalline crust of a dirty blue colour. The excess of acid is to be drained from the salt, and then it is to be washed with absolute alcohol. The salt gradually swells, and is reduced to a light crystalline powder of an ultramarine blue colour; it is then washed with alcohol, which it always renders of a blue colour, although but a very small quantity is dissolved. It is afterwards dried by being placed under a receiver, with a vessel containing snlphuric acid or chloride of calcium. An essential difference appears to exist between the salt which crystallizes in the concentrated acid and the blue pulverulent salt; but I do not know in what it consists. It is probable that the former is a supersalt; for the blue powder which I analysed is neutral sulphate. In this state sulphate of vanadium appears but slightly soluble in cold water; it first diffuses itself through it, and dissolves with extreme slowness, but in hot water it dissolves readily: the supersulphate is deliquescent in warm moist air, and becomes of a syrupy consistence; while the same quantity of sulphate, kept under water, remains almost entirely insoluble. It is rather difficult to obtain this salt in regular crystals.

The best method of crystallizing it is to let the dry sulphate deliquesce, and to suffer it to remain for some weeks. A very slight excess of acid often favours the experiment, which never succeeds in moist weather. The crystals consist principally of an aggregation of prisms; but I obtained some which were very short simple right prisms with rhombic bases, having small triangular oblique facets at the summit of each acute angle. Their colour resembles the fine blue of sulphate of copper, but is perhaps rather deeper. This salt contains 17.9 per cent. of water, the oxygen of which is to that of the base as 2 to $\mathrm{l}$. This also is the composition of the powder precipitated by alcohol. Sulphate of vanadium is decomposed by heat; the oxide is converted into vanadic acid at the expense of the sulphuric acid; sulphurous and anhydrous sulphuric acid are disengaged, and fused vanadic acid remains. If hydrate of vanadium be digested in a slightly concentrated solution of the sulphate, the bydrate is dissolved, 
and a soluble subsalt is obtained, which dries by spontaneous evaporation into a blue transparent varnish, which becomes brown and loses water when heated to $212^{\circ}$ Fahrenheit. The water redissolves it; but the solution exposed for a long time to the influence of the air becomes green, and the salt is eventually converted into vanadate of vanadium, which is deposited, and leaves the neutral blue salt in the state of a concentrated solution.

Sulphate of Vanadium and Potash. - This is obtained by mixing, in proper proportions, the solutions of the two neutral salts. The double salt does not crystallize, but dries into a gummy mass, of a light blue colour, which contains no trace of crystallization.

Nitrate of Vanadium.-Nitric acid dissolves vanadium, its oxide and suboxide; the solution has a blue colour, which is not altered by boiling: but when hydrate of vanadium is dissolved to saturation in nitric acid, and the solution is suffered to crystallize spontaneously, it becomes green when it has acquired a certain degree of concentration, and at the instant of complete desiccation the acid decomposes and leaves vanadic acid, which retains a small quantity of combined nitric acid.

Phosphate of Vanadium. - The neutral salt gives a blue syrup, which does not crystallize, and which, when dried with heat, becomes white and swells like alum dried in the fire. At a white heat it becomes round and agglomerates, but it does not fuse. It is then of a deep colour, and completely insoluble in water. The phosphate may be obtained in very small blue crystals by mixing it with a certain excess of phosphoric acid, and evaporating the solution at the temperature of $123^{\circ}$ Fahrenheit. After some time the neutral salt is found crystallized in the midst of a colourless mother-water, which is merely concentrated phosphoric acid; it may be afterwards removed by alcohol. In the air these crystals rapidly deliquesce; when a concentrated solution of phosphate of vanadium is mixed with absolute alcohol, a grayish blue gelatinous precipitate is formed, which when washed with alcohol and dried is almost white, and does not alter in the air. It does not completely dissolve in water, and appears to be a subsalt.

Arseniate of Vanadium.-A solution of this salt containing an excess of arsenic acid gives by evaporation a crust composed of small crystalline grains of a light blue colour, which may be deprived readily of the excess of acid by washing with water. It dissolves so very slowly even in hot water acidulated with arsenic acid, that it might be thought insoluble; water, nevertheless, is capable of holding much of it in solution. Muriatic acid dissolves the crystals readily. If arsenic 
acid be saturated with hydrate of vanadium, a very concentrated solution is obtained, which furnishes by evaporation a crystallized neutral salt, and also a gummy mass which appears to be a subsalt. Alcohol acts upon the arseniate in the same manner as upon the phosphate.

Borate of Vanadium.-A solution of borax precipitates the salts of vanadium of a brownish gray colour. This precipitate is dissolved by an excess of boracic acid. The solution is blue, but it soon becomes green in the air. If an attempt is made to restore the blue colour by passing a current of sulphuretted hydrogen into it, oxide of vanadium and subsulphuret are formed, which remain dissolved in the boracic acid, and colour the solution of an intense brown. Sulphuric acid quickly precipitates this solution, and the precipitate consists of subsulphuret of vanadium. Subjected to spontaneous evaporation in the air, the solution soon becomes green, and leaves at last a solid mixture, of a greenish brown colour, of sulphate and vanadate of vanadium, sulphur, and boracic acid in crystalline scales.

Carbonate of Vanadium.-It appears that this salt cannot be obtained in the moist way. I have stated above that the precipitate formed by the alkaline carbonates in the salts of vanadium consists of hydrate free from carbonic acid, or contains only traces of it; the presence of carbonic acid depends upon accidental circumstances, as happens with the oxides of cobalt and nickel.

Silicated Vanadium. - Prepared by double decomposition; this salt forms a greyish precipitate, which becomes green by drying. Water does not separate anything from the green powder.

Molybdate of Vanadium.-When sulphate of vanadium is mixed with molybdate of ammonia, both in solution, a liquid of a fine purple colour is obtained similar to that of tungstate of molybdenum. On this account I imagined that there was an exchange of oxygen, and that vanadate of molybdenum was formed. But when a molybdate is mixed with vanadate of ammonia, it becomes yellow and not purple. The purple colour disappears gradually in the air; it is at first replaced by blue, then by green, and lastly by yellow, without the solution becoming turbid.

Tingstate of Vanadium.-This salt is precipitated in the form of a brownish yellow powder. It dissolves in a sufficient quantity of water, and when it is left in the liquid it dissolves without the addition of water, in proportion as the oxide of vanadium acidifies. The solution eventually contains a yellow combination of the two acids. 
Chromate of Vanadium.-Chromic acid dissolves hydrate of vanadium. The solution is of a brownish yellow colour, and by spontaneous evaporation leaves a brown polished varnish, without any trace of crystallization. This varnish does not completely redissolve in water, and the fresh solution is yellow. Sulphuretted hydrogen precipitates a greenish mass, and the liquid becomes colourless.

Oxalate of Vanadinem. - Oxalic acid saturated with hydrate of vanadium, and evaporated, gives a blue varnish, which is transparent and dissolves slowly in cold water, but more readily in warm water. This salt mixed with oxalic acid gives a blue crystalline salt, which is very soluble in water. It remains to be determined whether the first is a neutral or subsalt, and whether the second is, as it appears to be, a supersalt.

Tartrate of Vanadium.-This salt is of a light blue colour, the remarkable beauty of which is derived from its not becoming green by contact with the air. In the dry state it forms a transparent splintery mass, which requires many hours for solution in cold water, but hot water dissolves it more readily. Ammonia dissolves it; the solution has a fine purple colour, which it loses by the acidification of the oxide of vanadium when it is exposed to the air.

Oxalate of Vanadium and Potash.-This salt gives a blue mass, which does not crystallize. Oxalic acid and the binoxalates decompose vanadic acid, and form blue salts of vanadium.

Tartrate of Vanadium and Potash. - This salt has the appearance of a splintery extractive mass; its colour is blue with a strong shade of violet. It may be obtained also by dissolving vanadic acid by means of bitartrate of potash : in this case part of the tartaric acid is clecomposed. Ammonia does not precipitate the double tartrate, but it imparts to it a magnificent purple colour, which is destroyed by the action of the air.

Citrate of Vanadium.-This salt is uncrystallizable, and gives a splintery mass of a very deep blue colour. It redissolves slowly in cold water; the solution is of a pure blue colour. Ammonia dissolves it, and assumes a yellowish brown colour, which gradually disappears by oxidation when exposed to the air.

Acetate of Vanadium. - Dilute acetic acid dissolves very little hydrate of vanadium. The liquid is of a pale blue colour, and yields a deposit when suffered to evaporate spontaneously; this is a white powder, whilst the excess of acid evaporates. When evaporated in a stove it becomes green, and the resi- 
duum is no longer soluble even in concentrated acetic acid. Acetate of potash does not precipitate the salts of vanadium. Concentrated acetic acid dissolves hydrate of vanadium, but the solution becomes green by spontaneous evaporation, and leaves a granular powder, composed of deep green opake microscopic crystals, the form of which is either a cube or a very short rectangular prism; they dissolve very slowly in water; the solution is of a deep green colour.

Formiate of Vanadium. - Artificial formic acid dissolves hydrate of vanadium, and gives by spontaneous evaporation a blue, opake, saline mass, which is easily soluble in cold water; but this solution, which contains no excess of water, becomes gradually green by contact with the air. The salt when perfectly dried is of a violet colour, with a tint of brown, and is not completely soluble in water.

Succinate of Vanadium.-Buccinic acid dissolves very little hydrate of vanadium; the solution is but slightly coloured. By evaporation the succinate is obtained in the state of a white powder, mixed with crystals of succinic acid. Nevertheless, the salts of vanadium are not precipitated by neutral succinates, but the mixture soon becomes greenish.

Benzoate of Vanadium. - A boiling solution of benzoic acid dissolves a little hydrate of vanadium; submitted to a slow evaporation the solution deposits a yellow powder, and the excess of acid crystallizes around it.

[To be continued.]

XLII. Particulars of the Measurement, by various Methods, of the Instrumental Error of the Horizon-Sector described in Phil. Mag. vol. lix. By JoHn Nrxon, Esq.

[Continued from page 96.]

By the Eleventh Method.

Theory.- ET E (next page) represent the eye end, and O the object-glass end of the (horizontal line) of collimation of a perfect telescopic-lerel (or of the horizon-sector) correctly adjusted for taking a level. If we increase the diameter of the cylindrical ring nearest the object-glass, by for instance coiling a quantity of thread about it, the line of collimation will be elevated in the direction FP; and on reversing the telescope within its $\mathbf{Y s}$, it will be equally elevated in the opposite direction, or $\mathrm{GQ}^{*}$; the angle $\mathrm{PEO}=\mathrm{QOE}$ being

* Admitting the $Y \mathrm{~s}$ to have the same angular opening; but it is evident from the demonstration given in page 429 of last volume, that were the Ys unequal the theory would be equally applicable.

N.S. Vol. 10. No. 59. Nov. 1831. 